\title{
Ampliação da distribuição de espécies de vespas sociais neotropicais no Brasil (Hymenoptera: Vespidae: Polistinae)
}

Apesar do crescente esforço para o conhecimento da diversidade de vespas sociais neotropicais que ocorreu na última década, ainda há, biomas, regiões e/ou estados subamostrados ou com ausência de listas de espécies. Nessa perspectiva foram realizadas coletas esporádicas em diferentes estados e ecossistemas brasileiros no período de 2013 a 2015. Esse esforço amostral resultou em novos registros para estado do Maranhão: Polistes subsericius Saussure, 1854; Ceará: Metapolybia servilis Cooper, 1999, sendo esta, até o momento desconhecida no Brasil; para o estado do Tocantins, todos os registros foram inéditos, uma vez que, o estado não apresenta listas de vespas sociais: Apoica pallida (Olivier, 1792), Apoica thoracica du Buysson, 1906, Apoica strigata Richards, 1978, Apoica arborea de Saussure, 1854, Mischocyttarus flavicornis Zikán, 1935 e Polybia striata (Fabricius, 1787).

Palavras-chave: Cerrado; Diversidade; Metapolybia; Mata de Cocais.

\section{Expansion of the distribution area of neotropical social wasp species in Brazil (Hymenoptera: Vespidae: Polistinae)}

Although the growing effort to understand the diversity of neotropical social wasps, that occurred in the last decade, there are still biomes, regions and/or states that are subsampled or with no list of species. In this perspective, sporadic collections were carried out in different Brazilian states and ecosystems in the period from 2013 to 2015. This sampling effort resulted in new records for the state of Maranhão: Polistes subsericius Saussure, 1854; Ceará: Metapolybia servilis Cooper 1999, this being, until now unknown in Brazil; for the state of Tocantins, all records were unpublished, since the state does not present lists of social wasps: Apoica pallida (Olivier, 1792), Apoica thoracica du Buysson, 1906, Apoica strigata Richards, 1978, Apoica arborea de Saussure, 1854, Mischocyttarus flavicornis Zikán, 1935 and Polybia striata (Fabricius, 1787).

Keywords: Cerrado; Diversity; Metapolybia; Mata de Cocais.

Topic: Conservação da Biodiversidade

Reviewed anonymously in the process of blind peer.

Marcos Magalhães de Souza

Instituto Federal do Sul de Minas Gerais, Brasil

http://lattes.cnpq.br/2334845279402555

http://orcid.org/0000-0003-0415-1714

marcos.souza@ifsuldeminas.edu.br

Epifânio Porfiro Pires

Universidade Federal de Lavras, Brasil

http://lattes.cnpq.br/9861269484139415

http://orcid.org/0000-0001-6950-3165

epifanioppires@gmail.com

Evando Luiz Coelho

Instituto Federal do Sul de Minas Gerais, Brasil

http://lattes.cnpq.br/3466040624364426

http://orcid.org/0000-0001-6222-8703

ecoelho@ifce.edu.br
Received: 02/05/2020

Approved: 29/06/2020

\author{
Mateus Aparecido Clemente \\ Universidade Estadual Paulista "Júlio de Mesquita Filho", Brasil \\ http://lattes.cnpq.br/6924671025219857 \\ http://orcid.org/0000-0003-4969-1335 \\ mateus1981@gmail.com \\ Paloma Pereira Bonfitto ii \\ Instituto Federal do Sul de Minas Gerais, Brasil \\ http://lattes.cnpq.br/7771011160112984 \\ http://orcid.org/0000-0003-4644-1023 \\ palomapbonfitto@gmail.com
}

Referencing this:

SOUZA, M. M.; PIRES, E. P.; COELHO, E. L.; CLEMENTE, M. A.; BONFITTO, P. P.. Ampliação da distribuição de espécies de vespas sociais neotropicais no Brasil (Hymenoptera: Vespidae: Polistinae). Nature and Conservation, v.13, n.3, p.1-6, 2020. DOI: http://doi.org/10.6008/CBPC2318-2881.2020.003.0001 


\section{INTRODUÇÃO}

As vespas sociais (Hymenoptera, Vespidae) são insetos conhecidos popularmente como marimbondos ou cabas (SOUZA et al., 2012), possuem distribuição cosmopolita, com a maior diversidade de espécies na região Neotropical (CARPENTER, 1981; CARPENTER et al., 2001). No Brasil, os representantes desse grupo pertencem à subfamília Polistinae, distribuídas em três tribos: Mischocyttarini, Polistini e Epiponini (CARPENTER et al., 2001; CARPENTER, 2004).

Apesar do crescente esforço para o conhecimento da diversidade de vespas sociais na região neotropical, coletas são escassas ou mesmo ausentes em muitas regiões e ecossistemas, como as Matas de Cocais no estado do Maranhão, Cerrado em Tocantins e Caatinga no estado do Ceará (RICHARDS, 1978; ANDENA et al., 2014; SOUZA et al., 2015; BARBOSA et al., 2016; SOUZA et al., 2020).

Para o estado do Maranhão há informações da fauna de Polistinae produzidas por três estudos (RICHARDS, 1978; SILVA et al., 2011; SOMAVILLA et al., 2014), para o Ceará há compilados de dados (RICHARDS, 1978; ANDENA et al., 2014), e não há informações para o estado de Tocantins. No intuito de diminuir a lacuna de conhecimento sobre a distribuição geográfica da fauna de vespas sociais na região neotropical, o presente trabalho teve como objetivo apresentar novos registros de ocorrência e ampliação de áreas de distribuição geográfica de oito espécies para o Brasil.

\section{MATERIAS E MÉTODOS}

Foram realizadas coletas esporádicas no período de 2013 a 2015 nos estados de Tocantins, em áreas de Cerrado, nos municípios de Palmas ( $\left(0^{\circ} 11^{\prime} 4^{\prime \prime} \mathrm{S}, 48^{\circ} 20^{\prime} 1^{\prime \prime} \mathrm{W}\right)$, Ponte Alta do Tocantins $\left(10^{\circ} 44^{\prime} 38^{\prime \prime} \mathrm{S}, 47^{\circ}\right.$ $32^{\prime} 9^{\prime \prime}$ W) e Santa Tereza ( $\left(10^{\circ} 16^{\prime} 55^{\prime \prime} \mathrm{S}, 47^{\circ} 48^{\prime} 28^{\prime \prime}\right.$ W); no Ceará em 2014, na região de Caatinga no município de Missão Velha ( $\left.7^{\circ} 15^{\prime} 0^{\prime \prime} \mathrm{S}, 39^{\circ} 8^{\prime} 34^{\prime \prime} \mathrm{W}\right)$; em área de cultivo comercial de banana no Maranhão em 2014, no município de Codó ( $4^{\circ} 27^{\prime} 18^{\prime \prime} \mathrm{S}, 43^{\circ} 53^{\prime} 9^{\prime \prime} \mathrm{W}$ ) em áreas de Mata de Cocais (Figura 1).

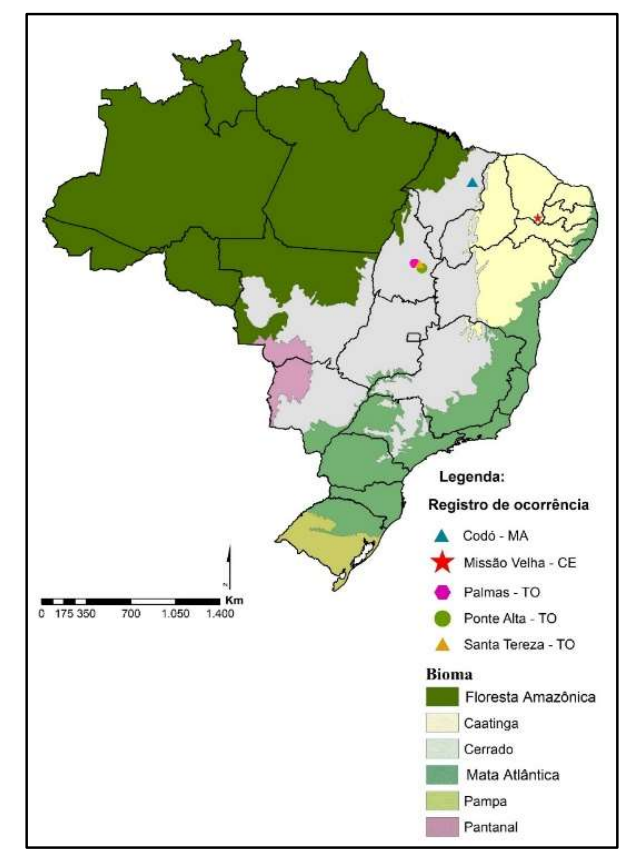

Figura 1: Localização das áreas de registro. 
O método de amostragem foi o de busca ativa com o auxílio de rede entomológica (SOUZA et al., 2006). A identificação das espécies de vespas sociais foi realizada pelo Dr. Orlando Tobias da Silveira do Museu Emílio Goeldi, Pará, e pelo Dr. Sergio Andena da Universidade Estadual de Feira de Santana, Bahia. Os exemplares encontram-se incorporados na coleção CBVS, sistema speciesLink, laboratório de zoologia do IFSULDEMINAS, Campus Inconfidentes.

\section{RESULTADOS E DISCUSSÃO}

Foram realizados novos registros, para o estado do Maranhão: Polistes subsericius Saussure, 1854; e outro para o Ceará: Metapolybia servilis Cooper, 1999, sendo esta, desconhecida até o momento para o Brasil. Para o estado de Tocantins, são apresentados os primeiros relatos sobre a composição da fauna de vespas sociais, devido ausência de estudos nessa região: Apoica pallida (Olivier, 1792), Apoica thoracica du (Buysson, 1906), Apoica strigata (Richards, 1978), Apoica arborea (Saussure, 1854), Mischocyttarus flavicornis Zikán, 1935 e Polybia striata (Fabricius, 1787) (Figura 2).

De acordo com a distribuição dessas espécies no Brasil, Apoica pallida tem registro para os estados do Acre, Amapá, Amazonas, Ceará, Goiás, Maranhão, Mato Grosso, Pará, Rondônia, Roraima, São Paulo (PICKETT et al., 2007) e Bahia (SILVA NETO et al., 2011). Apoica thoracica foi registrada nos estados do Acre, Amapá, Amazonas, Distrito Federal, Espírito Santo, Goiás, Mato Grosso, Minas Gerais, Pará, Paraná, Rondônia, Roraima e São Paulo (RICHARDS, 1978; PICKETT et al., 2007). Apoica strigata obteve registros nos estados do Acre, Amazonas, Maranhão, Mato Grosso e Pará (RICHARDS, 1978; PICKETT et al., 2007). Apoica arborea no Acre, Amazonas, Goiás, Maranhão, Mato Grosso, Pará e Rondônia (RICHARDS, 1978; PICKETT et al., 2007).

O gênero Mischocyttarus de Saussure compreende 253 espécies descritas com distribuição cumulativa se estendendo do sudeste do Canadá até o norte da Argentina (CARPENTER et al., 2001; SILVEIRA, 2008; BORGES et al., 2019). No Brasil são registradas cerca de 124 espécies (SILVEIRA, 2008; BORGES et al., 2019). Mischocyttarus flavicornis foi registrado no Brasil para os estados de Goiás, Maranhão, Mato Grosso, Pará (RICHARDS, 1978), Acre, Amazonas e Rondônia (SILVEIRA, 2013), sendo agora sua distribuição ampliada para o estado de Tocantins.

Polybia Lepeletier é um gênero com 58 espécies válidas que se estendem do sul dos Estados Unidos até o norte da Argentina (RICHARDS, 1978; CARPENTER et al., 2001). No Brasil já foram registradas 44 espécies, das quais três são endêmicas (CARPENTER et al., 2001). A ocorrência de Polybia striata foi registrada nos estados do Acre, Amazonas, Paraná, Espírito Santo, Goiás, Maranhão, Mato Grosso, Pará, Rio de Janeiro, Rondônia e São Paulo (RICHARDS, 1978).

Para o estado do Tocantins, sugere-se que, prioritariamente trabalhos de diversidade sejam realizados, pois até o presente momento não há registro de nenhum estudo no estado. $O$ trabalho de Richards (1978) menciona 62 espécies para o estado de Goiás, das quais, três são registradas no presente estudo (A. thoracica, $M$. flavicornis e $P$. striata). Mesmo levando em consideração os registros de ocorrência de espécies já existentes para o estado de Goiás segundo Richards (1978), A. arborea, A. strigata e Apoica 
pallida são relatadas pela primeira vez no presente estudo. A criação do estado de Tocantins foi efetivamente reconhecida a partir do dia 1ำ de janeiro de 1989. Após 26 anos de sua criação, o estado não apresenta informações sobre a fauna de vespas sociais (RICHARDS, 1978), o que faz desse trabalho a primeira lista de espécie de vespas sociais.
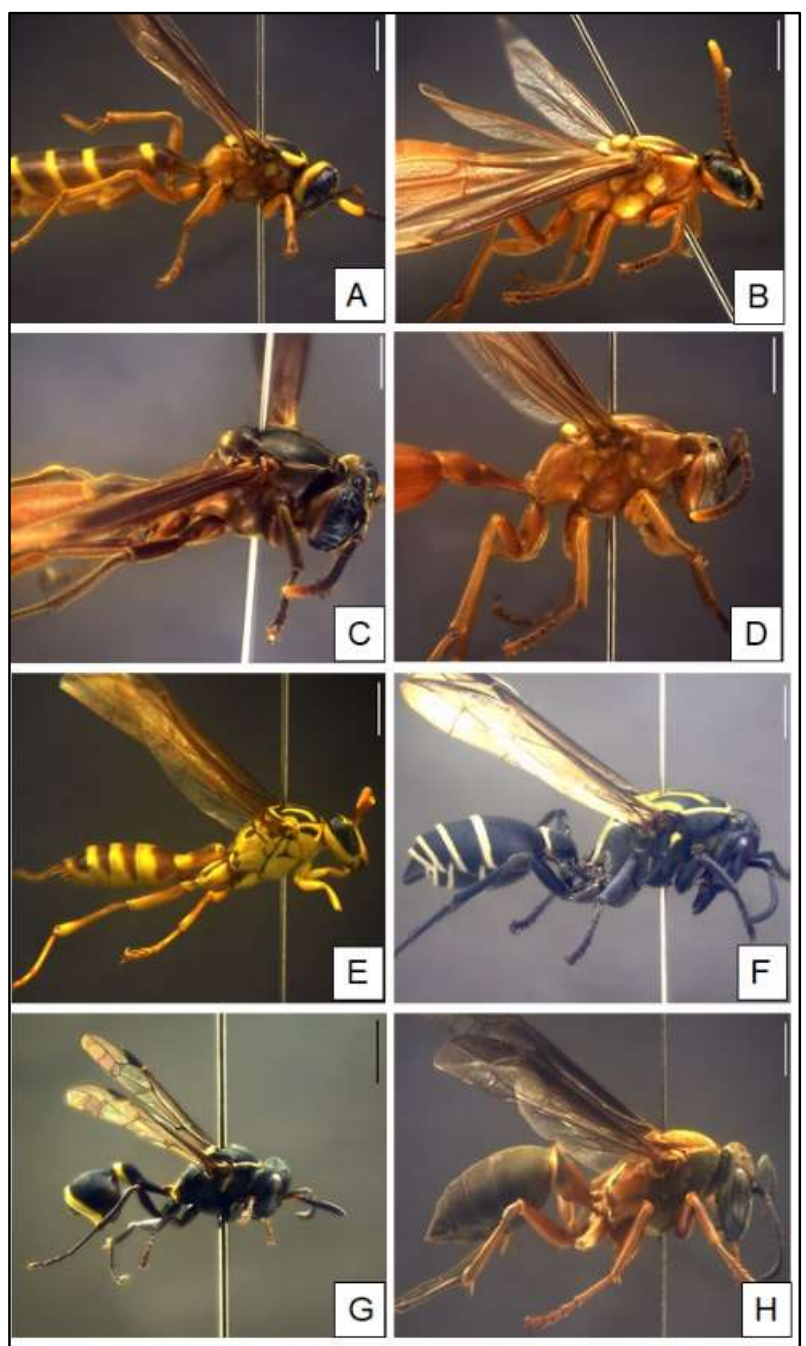

Figura 2: A: Novos registros de polistinae para o estado de Tocantins: Vista lateral de Apoica arborea; B: Vista lateral de Apoica pallida; C: Vista lateral de Apoica thoracica; D: Vista lateral de Apoica strigata; E: Vista lateral de Mischocyttarus flavicornis; F: Vista lateral de Polybia striata; Para o estado do Ceará G: Vista lateral de Metapolybia servilis; Para o estado do Maranhão: H: Vista lateral de Polistes subsericius.

O gênero Polistes Latreille é cosmopolita e apresenta mais de 200 espécies descritas, sendo a maioria delas de ocorrência tropical. No Brasil são registradas 38 espécies, das quais 10 são endêmicas (CARPENTER et al., 2001). Polistes subsericius é registrado pela primeira vez para o estado do Maranhão. No Brasil, essa espécie tem registro de ocorrência para os estados do Amazonas, Amapá, Bahia, Minas Gerais, Pará, Rio de Janeiro e São Paulo (RICHARDS, 1978). O registro dessa espécie amplia para 73 espécies com registros confirmados para o estado (RICHARDS, 1978; SILVA et al., 2011; SOMAVILLA et al., 2014).

Durante o presente estudo, registrou-se uma colônia de Metapolybia servilis Cooper, 1999, no semiárido cearense, sendo este o primeiro registro para o Brasil, ampliando para dez o número de espécies listadas para o país (ANDENA et al., 2014; ANDENA et al., 2011). A espécie Metapolybia servilis tinha registro de ocorrência apenas para o Paraguai e Costa Rica (COOPER, 1999), até o momento. 
Os estados do Ceará e Maranhão possuem poucas áreas amostradas e suas faunas são pouco conhecidas. À medida que novos estudos nesses estados sejam realizados, o número de espécie deve aumentar (SILVA et al., 2011; ANDENA et al., 2014; SOMAVILLA et al., 2014). Para o Maranhão, Silva et al. (2011) e Somavilla et al. (2014) discutem a importância da utilização de diferentes métodos de coleta, os quais podem revelar um número maior de espécies nas áreas estudadas, pois em geral, as espécies possuem um comportamento de forrageio variado.

\section{CONCLUSÕES}

Nas duas últimas décadas houve um expressivo número de publicações de inventários de vespas sociais em diferentes biomas e regiões do Brasil, sobretudo no estado de Minas Gerais. No entanto, esse número ainda é insuficiente para muitos estados, como Tocantins, onde não havia nenhum estudo, portanto é apresentada a primeira lista de vespas sociais para esse estado, e adicionalmente ampliou-se o número de espécies conhecidas no Brasil, com o registro de Metapolybia servilis, no estado do Ceará.

AGRADECIMENTOS: Ao Instituto Federal do Maranhão, Campus Codó.

\section{REFERÊNCIAS}

ANDENA, S. R.; CARPENTER, J. M.. A New Species of Metapolybia (Hymenoptera: Vespidae; Polistinae, Epiponini) Entomologica Americana, New York, v.117, n.3, p.117-120, 2011. DoI: https://doi.org/10.1664/11-RA-003.1

ANDENA, S. R.; CARPENTER, J. M.. Checklist das espécies de Polistinae (Hymenoptera, Vespidae) do semiárido brasileiro In: BRAVO, F.; CALOR, A.. Artrópodes do Semiárido: Biodiversidade e Conservação. Feira de Santana: Print Mídia, 2014. p.169-180

BARBOSA, B. C.; DETONI, M.; MACIEL, T.; PREZOTO, F.. Studies of social wasp diversity in Brazil: Over 30 years of research, advancements and priorities. Sociobiology, Feira de Santana, v.63, n.3, p.858-880, 2016. DOI: http://dx.doi.org/10.13102/sociobiology.v63i3.1031

BORGES, R. C.; SILVEIRA, O. T.. Revision of the species-group of Mischocyttarus (Omega) filiformis (de Saussure 1854), with description of three new species (Hymenoptera, Vespidae). Zootaxa, v.4657, p.545-564, 2019. DOI: http://dx.doi.org/10.11646/zootaxa.4657.3.7

CARPENTER, J. M.. The phylogenetic relationships and natural classification of the Vespoidea (Hymenoptera). Systematic Entomology, London, v.7, n.11-38, 1981. DOI: http://dx.doi.org/10.1111/j.1365-3113.1982.tb00124.x

CARPENTER, J. M.; MARQUES, O. M.. Contribuição ao estudo dos vespídeos do Brasil (Insecta, Hymenoptera, Vespoidea, Vespidae). Publicações Digitais, v.2, p.1-147, 2001.

CARPENTER, J. M.. Synonymy of the genus Marimbonda Richards, 1978, with Leipomeles Mobius, 1856 (Hymenoptera: Vespidae: Polistinae), and a new key to the genera of paper wasps of the new world. Americam
Museum Novitates, Nova York, v.3456, p. 1-16, 2004. DOI: http://dx.doi.org/10.1206/00030082(2004)465<0001:SOTGMR>2.0.CO;2

COOPER, M.. New species of Metapolybia Ducke (Hym., Vespidae, Polistinae). The Entomologists Monthly Magazine, London, v.135, p.107-110, 1999.

PICKETT, K. M.; WENZEL, J. W.. Revision and Cladistic Analysis of the Nocturnal Social Wasp Genus, Apoica Lepeletier (Hymenoptera: Vespidae; Polistinae, Epiponini). Americam Museum Novitates, Nova York, v.352, p.1-30, 2007.

RICHARDS, O. W.. The social Wasps of the Americas, Excluding the Vespinae. London: British Museum, Natural History, 1978.

SILVA NETO A. M.; ANDENA, S. R.. New records of Apoica pallida (Olivier, 1792) (Hymenoptera: Vespidae, Epiponini) in Bahia State. Entomo Brasilis, Vassouras, v.4, n.3, p.152153, 2011. DOI: http://dx.doi.org/10.12741/ebrasilis.v4i3.171

SILVA, S. S.; AZEVEDO, G. G.; SILVEIRA, O. T.. Social wasps of two Cerrado localities in the northeast of Maranhão state, Brazil (Hymenoptera, Vespidae, Polistinae). Revista Brasileira de entomologia, Curitiba, v.55, n.4, p.597-602, 2011. DOI: http://dx.doi.org/10.1590 / S008556262011000400017

SILVEIRA, O. T.. Phylogeny of wasps of the genus Mischocyttarus de Saussure (Hymenoptera, Vespidae, Polistinae). Revista Brasileira de Entomologia, Curitiba, v.52, n.4, p.510-549, 2008. DOI: http://dx.doi.org/10.1590/S008556262008000400004 
SILVEIRA, O. T.. Social wasp species of Mischocyttarus (Phi) related to $M$. alfkenii (Ducke) and M. paraguayensis Zikán (Hymenoptera, Vespidae, Polistinae). Revista Brasileira de Entomologia, Curitiba, v.57, p.173-196, 2013. DOI: http://dx.doi.org/10.1590/S0085-56262013005000017

SMETHURST, M.; CARPENTER, J. M.. A new species of Metapolybia Ducke from Central America (Hymenoptera: Vespidae; Polistinae). Journal of the New York
Entomologícal Society, New York, v.105, p.180-185, 1998. DOI: http://dx.doi.org/10.5281/zenodo.26301

SOMAVILLA, A.; MARQUES, D. W.; BARBOSA, E. A. S.; PINTOJUNIOR, J. S.; OLIVEIRA, M. L.. Vespas Sociais (Vespidae: Polistinae) de uma Área de Floresta Ombrófila Densa Amazônica no Estado do Maranhão, Brasil. Entomo Brasilis, Vassouras, v.7, n.3, p.183-187, 2014. DOI: http://dx.doi.org/10.12741/ebrasilis.v7i3.404

A CBPC - Companhia Brasileira de Produção Científica (CNPJ: 11.221.422/0001-03) detém os direitos materiais desta publicação. Os direitos referem-se à publicação do trabalho em qualquer parte do mundo, incluindo os direitos às renovações, expansões e disseminações da contribuiç̃ão, bem como outros direitos subsidiários. Todos os trabalhos publicados eletronicamente poderão posteriormente ser publicados em coletâneas impressas sob coordenação da Sustenere Publishing, da Companhia Brasileira de Produção Científica e seus parceiros autorizados. Os (as) autores (as) preservam os direitos autorais, mas não têm permissão para a publicação da contribuição em outro meio, impresso ou digital, em português ou em tradução. 